\title{
Control and Optimization of Multi-Agent Systems and Complex Networks for Systems Engineering
}

\author{
Manuel Herrera ${ }^{1, *,+} \mathbb{D}$, Marco Pérez-Hernández ${ }^{1,+} \mathbb{D}$, Ajith Kumar Parlikad ${ }^{1,+} \mathbb{D}$ and Joaquín Izquierdo ${ }^{2,+} \mathbb{D}$ \\ 1 Institute for Manufacturing, Department of Engineering, University of Cambridge, 17 Charles Babbage Road, \\ Cambridge CB3 0FS, UK; mep53@cam.ac.uk (M.P.-H.); aknp2@cam.ac.uk (A.K.P.) \\ 2 Fluing-Institute for Multidisciplinary Mathematics (IMM), Universitat Politècnica de València, \\ Cno. de Vera s/n, 46022 Valencia, Spain; jizquier@upv.es \\ * Correspondence: amh226@cam.ac.uk \\ $\dagger$ These authors contributed equally to this work.
}

Citation: Herrera, M.; Pérez-Hernández, M.; Parlikad, A.K. Izquierdo, J. Control and Optimization of Multi-Agent Systems and Complex Networks for Systems Engineering. Processes 2021, 9, 2070. https://doi.org/10.3390/pr9112070

Received: 13 November 2021 Accepted: 15 November 2021 Published: 18 November 2021

Publisher's Note: MDPI stays neutral with regard to jurisdictional claims in published maps and institutional affiliations.

Copyright: (c) 2021 by the authors. Licensee MDPI, Basel, Switzerland. This article is an open access article distributed under the terms and conditions of the Creative Commons Attribution (CC BY) license (https:// creativecommons.org/licenses/by/ $4.0 /)$
Systems engineering crosses multiple engineering disciplines for the design, control, and overall management of engineered systems. Such systems can be classified into chemical, civil, management, electrical, mechanical, and geotechnical areas. All these systems have an intrinsic complexity such that their analysis and synthesis should be addressed through understanding the systems as a whole, as well as the interactions between their parts. Today, advancements in systems engineering are directly related to the latest disruptive technology developments, ranging from the Internet of Things (IoT) and cyber-physical systems to the creation of digital twins. Depending on the working areas, the applications are disparate, encompassing self-driving cars, food processing, business services, and infrastructure management, among many others. Systems engineering is a development framework for dynamic modelling and simulation, operation and control, optimization methods, and risk analysis related to all the aforementioned applications and working areas.

A system engineering process involves various levels of abstraction of a system at a multiple-resolution degree. Such processes are carried out at different parts, collective and individual, of the system, whose dynamics are the result of their own evolution along with the influence from other interconnected parts. System processes, then, work towards the optimization of individual and local (subsystem) objectives that lead to an optimal general performance. To this end, each individual component can be modelled as an agent that can be understood as autonomous learning units able to make locally informed decisions. Such agents can work collectively in a multi-agent system (MAS) to enhance its performance and capability of problem solving at multiple scales. The agents can be associated with the nodes of a complex network, in which the associated links model the communication between agents and/or their interconnections. Complex networks can also be associated as a representation of any technological or physical system in which any dynamical process actually occurs. Thereby, both complex networks and MAS are of pivotal importance for systems engineering and management.

This Special Issue, "Control and Optimization of Multi-Agent Systems and Complex Networks for Systems Engineering", can be viewed as a significant contribution to the systems engineering literature since it curates novel advances in theoretical developments and applications of biological and nature-inspired multi-agent and network models for industrial and engineering applications [1]. This Special Issue is timely since it comes as a methodological counterpart to the surge in disruptive technologies—such as cyber-physical systems, digital twins, and blockchain-for the operation, management and control of industrial and engineering systems processes.

\section{Overview of This Special Issue}

This Special Issue contains 11 papers which focus on some of the mentioned paradigms on MAS and complex networks for systems engineering. Central to this collection is a 
literature review on multi-agent systems and complex networks for engineering systems and processes [1]. The review introduces explanations and links to key bibliographic elements, as well as provides a critical view of state-of-the-art methods and technologies for the ongoing and future work in the area. This review presents theoretical aspects comprehensibly and in a well-structured manner, presenting several applications as an illustration of the usefulness of the contents.

The work of Meirelles et al. [2] focuses more on a theoretical aspect of systems and optimization. Particularly, they present a novel swarm optimization procedure named "grand tour algorithm" (GTA), based on a cyclist peloton (similar in some respects to other swarms such as birds flying in formation). This paper shows how GTA exhibits a fairly good performance in terms of consistency and speed for the benchmarking of standard problems when evaluating optimization algorithms. GTA also showed an excellent scalability, being able to handle benchmarking problems of up to 20,000 variables.

The rest of the papers of this Special Issue can be classified into three key areas: (i) infrastructure management [3,4]; (ii) manufacturing and industrial processes [5-8]; and (iii) energy production processes [9-11].

\subsection{Infrastructure Management}

Two papers of this Special Issue revolve around infrastructure management, addressing challenges such as optimal vehicle routing and supply chain management. Kovaks et al. [3] present a tool for vehicle routing algorithms, named the "fitness landscape". Said tool aims to analyse the search parameters for relevant NP-hard algorithms such as the vehicle routing, having applications in logistics processes and other road infrastructure challenges. The way in which the fitness landscape works is by searching the parameter space and tuning the parameters in an efficient and computationally optimal manner. The results, tested in the vehicle routing problem, showed that the fitness landscape benefits the algorithm performance when it is compared to other algorithms used for similar purposes.

Mzougi et al. [4] examined supply chain risks related to the automotive industry. The analyses are complemented by an innovative procedure for computing the risk priority number within a failure mode, effects and criticality analysis framework. The proposal has the additional benefit of using fuzzy numbers to better model the uncertainty associated with the problem.

\subsection{Manufacturing and Industrial Processes}

Four papers refer to the classification of manufacturing and industrial processes. Qian et al. [5] investigated a hybrid refrigeration system, with alternating and continuous currents, for food-handling purposes. Particularly, the authors explored the case study of oyster refrigeration. An important advantage of this proposal is that the refrigeration system was able to use both solar power and electricity to run, which could represent a sustainable solution when working on a larger scale.

The main aim of the paper by Abed et al. [6] is to reduce failures in manufacturing processes by analysing the production of associated waste. The analyses are conducted through the adoption of IoT technologies coupled with deep learning methods. This paper showed the results of a case study in painting bathtub backs (spraying sector). This manufacturing process presents defects (bubbles) due to alterations in the liquid paint mixture on a product surface during the aspiration process. The results are a significant improvement with respect to the reliability and control of the process, as well as with respect to the quality of the final product.

Bashir et al.'s [7] work focused on Petri net models for a global supervisory structure design, aiming to reduce the costs and complexities of manufacturing processes. This paper proposes two algorithms. The first is based on the concept of decentralized control for flexible manufacturing systems. This procedure uses a Petri net model to work by control zones. The second uses a centralised controller to supervise the system that may be 
decomposed at the zone levels computed by the first algorithm. The advantages of such a combination of algorithms were tested through two instances of flexible manufacturing designs. The advantages are the ability to decompose a suitable problem into subproblems (scalability), adequate computational complexity for the centralised controller, and the possibility of finding a minimum number of decentralised controllers for an optimal system performance.

Chen et al. [8] explored direct chill casting technology to produce aluminium alloys. They proposed a solution algorithm based on a transient study of grain structure and macrosegregation with a cellular automaton and finite element model. After a detailed description and numerical validation of the model, this paper presents results on temperature, grain structure, fluid flow, and macro-segregation for experiments with a grain-refined billet. The impact of the casting speed, casting temperature, and flow intensity were also investigated and could be used as guidance for pursuing similar experiments in the future.

\subsection{Energy Production Processes}

Three papers concern energy production processes. First, Izquierdo-Pérez et al. [9] investigate wind energy production. The authors developed the mathematical formulation of cost and decision functions and associated constraints to then run a particle swarm optimization (PSO) algorithm. This integrated tool aimed to minimise the average total cost of the energy of a wind farm (25 platforms and a grid connection) during a certain time span. After a sensitivity analysis over the tool parameters, the authors found that after an optimal selection of the model inputs it is possible to reach a cost reduction of over the $70 \%$ of the initial costs.

The work of Ridha et al. [10] is about solar energy. Particularly, the authors proposed a multi-objective PSO algorithm and two variants, adaptive weights PSO and sigmoid function PSO, to optimise the sizing of the stand-alone photovoltaic (PV) systems. The associated costs were related to the loss of load probability, life cycle cost, and levelised cost of energy. The presented results are for the case study of Kuala Lumpur, Malaysia, showing a better performance associated with the sigmoid function variant of PSO. In another work, Ridha et al. [11] optimally sized a PV system considering technological and economical objectives. The innovative, hybrid optimization procedure starts with an estimation of the initial values for a number of PV modules and battery capacity. This initial solution evolves to an optimal configuration in an iterative procedure, generating a set of optimal solutions through a modified version of the Pareto front. The process ended by running a ranking algorithm, based on an analytic hierarchy process, for the final solutions. A case study of Subung Jaya, Klang Valley, Malaysia, was used to show the results on the optimal number of PV modules and battery capacity.

\section{Conclusions}

This Special Issue, "Control and Optimization of Multi-Agent Systems and Complex Networks for Systems Engineering", presents a number of powerful techniques able to cope with the complexity associated with systems engineering processes, modelling and analysis. Agent-based models, such as bio-inspired, distributed and evolutionary algorithms, play an important role in systems engineering processes as agents are naturally able to model the dynamics of any system element or subsystem. In addition, these algorithms, by themselves, have shown their high usefulness in solving complex optimization processes using a decentralised approach. A problem approach combining complex networks with agent-based models reaches a deep understanding of engineering systems and dynamics, aiding their modelling and analysis further.

Overall, this Special Issue encompasses a collection of proposals that, considering the used methodology, can be classified as follows:

- Optimization, both classical and evolutionary;

- Swarm algorithms, variations of well-known algorithms, such as PSO, as well as other innovative procedures, such as the GTA algorithm; 
- General agent-based systems;

- Petri net models;

- Network dynamics and other complex networks methods for problem modelling and analysis;

- Machine learning methods, including (deep) neural networks.

This Special Issue also comprises novel theoretical developments and a range of applications. With respect to the applications, the papers herein can be classified as follows:

- Infrastructure management: vehicle routing, supply chain, and risk management;

- Manufacturing and industrial processes: sustainability, food chain, energy efficiency, flexible design, and production processes;

- Energy production processes: hybrid wind-wave farms, PV models, and battery capacity.

Contributions to this Special Issue, exploring new research avenues on multi-agent systems and complex networks, are expected to be of great value for academia, systems engineers practitioners, and asset managers. Furthermore, it highlights the importance of most of the applications presented with regard to the social and environmental benefits of a more sustainable and efficient use of infrastructure and industry resources.

Funding: This research was funded by the EPSRC and BT Prosperity Partnership project: Next Generation Converged Digital Infrastructure, grant number EP/R004935/1.

Conflicts of Interest: The authors declare no conflict of interest.

\section{References}

1. Herrera, M.; Pérez-Hernández, M.; Kumar Parlikad, A.; Izquierdo, J. Multi-Agent Systems and Complex Networks: Review and Applications in Systems Engineering. Processes 2020, 8, 312. [CrossRef]

2. Meirelles, G.; Brentan, B.; Izquierdo, J.; Luvizotto, E., Jr. Grand Tour Algorithm: Novel Swarm-Based Optimization for High-Dimensional Problems. Processes 2020, 8, 980. [CrossRef]

3. Kovács, L.; Agárdi, A.; Bányai, T. Fitness Landscape Analysis and Edge Weighting-Based Optimization of Vehicle Routing Problems. Processes 2020, 8, 1363. [CrossRef]

4. Mzougui, I.; Carpitella, S.; Certa, A.; El Felsoufi, Z.; Izquierdo, J. Assessing Supply Chain Risks in the Automotive Industry through a Modified MCDM-Based FMECA. Processes 2020, 8, 579. [CrossRef]

5. Qian, X.; Yang, Y.; Lee, S.W.; Caballes, M.J.L.; Alamu, O.S. Cooling Performance Analysis of the Lab-Scale Hybrid Oyster Refrigeration System. Processes 2020, 8, 899. [CrossRef]

6. Abed, A.M.; Elattar, S.; Gaafar, T.S.; Alrowais, F.M. The Neural Network Revamping the Process's Reliability in Deep Lean via Internet of Things. Processes 2020, 8, 729. [CrossRef]

7. Bashir, M.; Hong, L. Global Supervisory Structure for Decentralized Systems of Flexible Manufacturing Systems Using Petri Nets. Processes 2020, 7, 595. [CrossRef]

8. Chen, Q.; Li, H.; Shen, H. Transient Modeling of Grain Structure and Macrosegregation during Direct Chill Casting of Al-Cu Alloy. Processes 2020, 7, 333. [CrossRef]

9. Izquierdo-Pérez, J.; Brentan, B.; Izquierdo, J.; Clausen, N.E.; Pegalajar-Jurado, A.; Ebsen, N. Layout Optimization Process to Minimize the Cost of Energy of an Offshore Floating Hybrid Wind-Wave Farm. Processes 2020, 8, 139. [CrossRef]

10. Ridha, H.M.; Gomes, C.; Hizam, H.; Ahmadipour, M.; Halboot Muhsen, D.; Ethaib, S. Optimum Design of a Standalone Solar Photovoltaic System Based on Novel Integration of Iterative-PESA-II and AHP-VIKOR Methods. Processes 2020, 8, 367. [CrossRef]

11. Ridha, H.M.; Gomes, C.; Hizam, H.; Ahmadipour, M. Optimal Design of Standalone Photovoltaic System Based on MultiObjective Particle Swarm Optimization: A Case Study of Malaysia. Processes 2020, 8, 41. [CrossRef] 